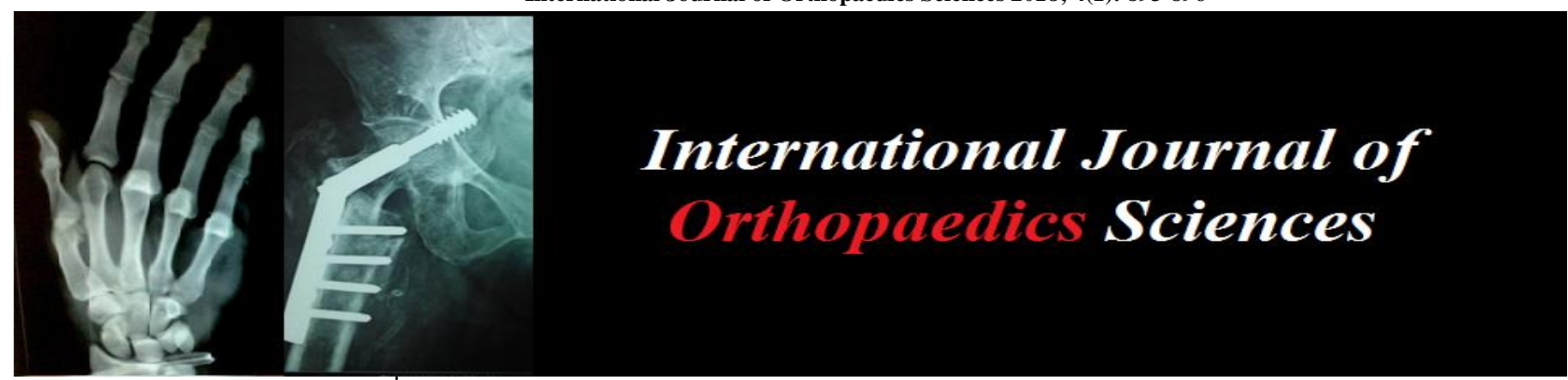

ISSN: $2395-1958$

IJOS 2018; 4(1): 893-896

(C) 2018 IJOS

www.orthopaper.com

Received: 15-11-2017

Accepted: 19-12-2017

Dr. Paramesha KC

Department of Orthopedics, MMC \& RI, Mysore, Karnataka, India

Dr. Rahul Bansal

Department of Orthopedics,

MMC \& RI, Mysore, Karnataka, India

Correspondence

Dr. Rahul Bansal

Department of Orthopedics,

MMC \& RI, Mysore, Karnataka,

India

\section{Functional outcome in patients with distal radius fractures treated with fragment specific fixation at tertiary care teaching hospital}

\section{Dr. Paramesha KC and Dr. Rahul Bansal}

DOI: $\underline{\text { https://doi.org/10.22271/ortho.2018.v4.i1m.130 }}$

Abstract

Introduction: Aim of this study was to study the functional outcome in patients with distal radius fractures treated with ORIF with $2.7 \mathrm{~mm}$ distal radius plates at 6 months using PRWE score. To study how early the patients can resume activities of their daily living and study various complications related to fragment specific fixation.

Methodology: It was a prospective study. All the patients underwent open reduction and internal fixation using fragment specific locking plates based on fracture patterns. Patients were followed up regularly up to 6 months post op. Functional outcomes were analyzed after 6 months of fixation using patient rated wrist evaluation score.

Results: In our study $84.38 \%$ patients were male (n 27) with average age being 38.59 years. Right side was the dominant wrist seen in 56\% patients (n 18). Most common mechanism of injury was to road traffic accidents. Average time from injury to surgery was 7.06 days and average PRWE score by the end of 6 months was 16.53 . Excellent outcomes were seen in $71.88 \%$ patients (n 23), good in $18.75 \%$ (n 6). Superficial infection, secondary collapse and stiffness and numbness were most common complications seen in 2 patients each. 31 patients had fracture union within 3 months and 30 patients returned to pre injury level of activities within 6 months.

Conclusion: We conclude from our data that among various fixation methods, fragment specific fixation provides stable and reliable fixation with good functional outcomes as shown by excellent PRWE scores.

Keywords: DER, Fragment specific fixation, PRWE score

\section{Introduction}

Fracture of the distal radius continues to be one of the most common skeletal injuries treated by Orthopaedics Surgeons. In fact these injuries account for approximately one sixth of all fractures seen and treated ${ }^{[1]}$. They constitute $17 \%$ of all fractures and $75 \%$ of all forearm fractures ${ }^{[2]}$.

Incidence of intra-articular comminuted Colles' is $23.8 \%$, chauffeur being $6.3 \%$, comminuted Smith is $2.5 \%$, volar barton has incidence of $1.3 \%$, combined dorsal barton and chauffeur comprises of $1.3 \%$, dorsal barton accounts for $0.5 \%$ and combined volar barton and chauffeur is rarest at $0.3 \%{ }^{[3]}$.

Fractures of the distal radius present a unique set of fixation issues and differ from most other sites. Distal fragments are periarticular and extremely small. The proximity to a tight network of tendons and retinacular sheaths preculedes the use of bulky plates. Distal fragments are too thin to provide adequate thread purchase for bony screws. The mere creation of holes for bone screws in distal fragments can result in iatrogenic comminution.

The last decade has witnessed an unprecedented interest in internal fixation techniques: First being columnar fixation with miniature or "fragment-specific" fixation ${ }^{[4]}$ and second fixation of a wide variety of fractures with fixed-angle devices through a single volar approach ${ }^{[5]}$. Improvements in imaging and recognition of unstable fracture fragments have enabled surgeons to customize their approach and identify and rigidly fix highly comminuted fractures with internal fixation, thereby enabling earlier resumption of wrist motion.

The evidence on treatment of wrist fractures accumulated in the last century suggests four principal goals of intervention (ARMS). 
There is both general consensus and scientific evidence that restoration of the anatomy of the distal radius is closely linked to restoration of function. Consequently, closed or operative management should seek to restore: Articular congruity (to reduce the wear of articular cartilage and degenerative changes), Radial alignment and length (to restore kinematics of the carpus and radioulnar joint), Motion (digits, wrist, and forearm to optimize return to functional activities), Stability (to preserve length and alignment until healing of the fracture).

In an attempt to minimize the morbidity of extensive surgical dissections associated with conventional dorsal plate fixation of distal radius fractures, Robert Medoff devised a hybrid technique of percutaneous wire and plate fixation designed to fix individual fracture fragments through several small incisions [6]. His "fragment-specific" classification defines articular fractures of the radius by recognition of five elemental fracture fragments that are present alone or in combination in every fracture. He divided distal radial fracture fragments into radial styloid, volar lip, dorsal radial, dorsal ulnar and die-punch components (intra articular). The technique involves the use of ultrathin modular implants that can be shaped to customize fixation for different fragment configurations and builds on the work of Rikli and Regazzoni by placing these implants strategically along the radial and intermediate columns and place screws according to fragments to maximize construct rigidity. The use of the term "fragment-specific fixation" does not refer to a particular implant type, but to the concept of the use of one, two or more low-profile implants and screws placed strategically along the columns of the distal radius to fix individual fracture fragments.

The purpose of the study is to assess the indication of open reduction and internal fixation by using concept of fragment specific fixation using $2.7 \mathrm{~mm}$ low profile distal radius plates 4 singularly or in combination with or without the use of $\mathrm{k}$ wires and strategic screw placement to study the functional outcomes in these patients. The current literature offers no evidence to support the use of internal fixation over external fixation for unstable distal radius fractures ${ }^{[7]}$. We are trying to assess if fragment specific fixation can provide better functional outcomes and help patients return to their daily routine activities at the earliest, especially in the younger age groups.

\section{Methodology}

Our study is a series of 32 adult patients with distal radial fractures treated with ORIF using fragment specific fixation. Sample size calculated based on the following: Proportion: $17 \%$, Level of significance $=5 \%$ and absolute allowable error $=15 \%$

The Descriptives procedure displays univariate summary statistics for several variables in a single table and calculates standardized values ( $\mathrm{z}$ scores). Variables can be ordered by the size of their means (in ascending or descending order), alphabetically, or by the order in which the researcher specifies.

Study Subjects: Study subjects were adult patients with fracture distal end of radius.

\section{Inclusion Criteria}

- Age group: 18 years and above Male and female patients.

- Intra-articular fracture distal end radius of either side or both with or without ulnar styloid fracture.

- Closed fractures.

- Fractures with history of trauma $<3$ weeks.

\section{Exclusion Criteria}

- Patients with comorbid conditions preventing surgical intervention Patients with local tissue condition making the surgery inadvisable Pathological fractures other than primary osteoporosis.

- Compound fractures

- Multiple fractures in the same limb

\section{Results}

Table 1: Age incidence

\begin{tabular}{|c|c|c|}
\hline Age in years & Number of Cases & Percentage \\
\hline$<25$ & 7 & 21.88 \\
\hline $26-35$ & 8 & 25 \\
\hline $36-45$ & 8 & 25 \\
\hline $46-55$ & 6 & 18.75 \\
\hline $56-65$ & 3 & 9.38 \\
\hline Total & 32 & 100 \\
\hline
\end{tabular}

It is inferred from table 1 that out of the total 32 patients, $21.88 \%$ were $<25$ years, $25 \%$ between $26-35$ years, $25 \%$ between $36-45$ years, $18.75 \%$ between $46-55$ years and $9.38 \%$ patients between 56-65 years. Maximum patients were between the age of 26 and 45 years.

Table 2: Sex incidence

\begin{tabular}{|c|c|c|}
\hline Sex & Number of cases & Percentage \\
\hline Male & 27 & 84.38 \\
\hline Female & 5 & 15.62 \\
\hline Total & 32 & 100 \\
\hline
\end{tabular}

It is evident from table 2 that out of total 32 patients, 27 $(84.38 \%)$ were males and $5(15.62 \%)$ female.

Functional results were assessed using patient rated wrist evaluation questionnaire. The questionnaire took into account patients rated pain score in various situations as well as functions based on usual activities and specific activities. Based on the PRWE scores patients were graded as excellent, good, fair and poor.

In our series out of 32 patients function was excellent in 23 $(71.88 \%)$ patients, good in $6(18.75 \%)$ cases, fair in $2(6.25 \%)$ patients and poor in only $1(3.12 \%)$ patient. Poor results were seen in patients who had secondary collapse and later went on to have stiffness and patients who did not follow rehabilitation protocol and thus had poorer results.

Table 3: Functional outcome based on PRWE score

\begin{tabular}{|c|c|c|}
\hline Functional outcome & Number of cases & Percentage \\
\hline Excellent & 23 & 71.88 \\
\hline Good & 6 & 18.75 \\
\hline Fair & 2 & 6.25 \\
\hline Poor & 1 & 3.12 \\
\hline Total & 32 & 100 \\
\hline
\end{tabular}

Range of movements were scored on a 26 point scale based on amount of dorsiflexion, palmarflexion, radial deviation, ulnar deviation, pronation and supination and final score were graded as being excellent, good, fair and poor.

In our series out of 32 patients, excellent range of movements were seen in $20(62.5 \%)$ patients, good range was seen in 9 $(28.12 \%)$ cases, fair movements in $1(3.13 \%)$ cases and poor range of movements in $2(6.25 \%)$ patients.

The mean wrist motion was as follows: $59.22^{\circ}$ of extension (range, $10-75^{\circ}$ ), $65.10^{\circ}$ of flexion (range, $30-82^{\circ}$ ), 28.94 of 
ulnar deviation (range, $15-38^{\circ}$ ), $17^{\circ}$ of radial deviation (range, $8-22^{\circ}$ ), $72.35^{\circ}$ of pronation (range, $50-83^{\circ}$ ), and $75.5^{\circ}$ of supination (range, $30-90^{\circ}$ ).

Table 4: Outcome based on range of movements

\begin{tabular}{|c|c|c|}
\hline Range of movements & Number of cases & Percentage \\
\hline Excellent & 20 & 62.5 \\
\hline Good & 9 & 28.12 \\
\hline Fair & 1 & 3.13 \\
\hline Poor & 2 & 6.25 \\
\hline Total & 32 & 100 \\
\hline
\end{tabular}

Two patients had superficial infection which responded well to a course of antibiotics and excellent outcomes.

Secondary collapse of fracture with stiffness: This was seen in two patients due to inappropriate load bearing and mobilization and they were then immobilized for longer periods leading to stiffness. They went on to have fair to poor outcomes and needed secondary procedures.

Tingling and numbness: Transient tingling and numbness was observed in two patients who were given $75 \mathrm{mg}$ of Pregabalin once a day and this recovered within 8 weeks.

Tourniquet palsy: One patient had tourniquet palsy which recovered well within 12 weeks. The patient went on to have excellent functional outcome with good range of movements. Persistent pain: One patient complaint of persistent pain which was managed by giving adequate analgesics.

Table 5: Complications

\begin{tabular}{|c|c|c|}
\hline Complications & Number of cases & Percentage \\
\hline Superficial infection & 2 & 6.25 \\
\hline Secondary collapse and stiffness & 2 & 6.25 \\
\hline Tingling and numbness & 2 & 6.25 \\
\hline Tourniquet palsy & 1 & 3.12 \\
\hline Persistent pain & 1 & 3.12 \\
\hline
\end{tabular}

\section{Discussion}

The distal radius fracture was first described by Abraham Colles in 1814. The fractures of distal end radius are most common fractures of upper extremity encountered in practice and constitutes $17 \%$ of all fractures and $75 \%$ of all forearm fractures. It is remarkable that this is one of the most challenging fracture to treat for the surgeon.

An anatomical reduction of the joint surface with rigid fixation is the main goal in the treatment of intra-articular fractures. It has been shown that residual intra-articular incongruity leads to posttraumatic arthritis in the long term. A better understanding of wrist anatomy and functioning through the studies conducted in the recent years as well as the increasing expectations of the patients have expanded the indications of surgical management.

The current trend of management for irreducible compression fractures of the joint surface ( $\mathrm{C} 3$ fractures, or pilon fractures of the distal radius) has shifted from bridging external fixation, pins, and bone grafting to open reduction and stable internal fixation and functional after-treatment. Many studies show that the long-term functional results are equivalent, but the earlier functional results favor open reduction and internal fixation (ORIF) over external fixation.

Using the mini plating system helps in reduction of larger as well as smaller fragments, the fragments can be easily reduced under direct visualization and this helps in adequate reduction of articular fragment and minimizing the risk of future complications. Any of the surgical approaches can be used depending on the plate being utilized.
The present study was undertaken to assess the functional outcome of operative management of distal radial fractures by fragment specific fixation using $2.7 \mathrm{~mm}$ mini plates.

In our study we used PRWE scoring system to assess the functional outcomes. DASH score and PRWE are the most widely used scoring systems for assessment of fracture distal end radius. Other scoring systems that we come across literature are visual analogue scale, Gartland and Werley scores, Green and Obrein score and many more. But most widely accepted system is DASH scoring system. PRWE score is very simple and specific to the wrist, it reduces questionnaire fatigue. PRWE scores have excellent correlation with DASH scores thus making PRWE scores a good scoring system for assessing functional outcome in our study ${ }^{[8]}$.

In our study distal end radius fractures were more common in 3rd to 5th decades with average age being 38.59 years. Most cases in our study were in younger patients requiring surgery after a high energy trauma and RTA. Fractures in older patients were mostly due to fall on an outstretched hand most of extra articular fractures could be managed conservatively but intra articular fractures were operated.

The average age of patients in our study was 38.59 years which is similar to studies by Saw et al., and Mandalia et al. who had average age of 32.3 and 39.27 years respectively ${ }^{[9,}$ 10].

In our study the major complication included tingling and numbness and superficial infections in two patients, other complications included stiffness of joint with secondary collapse in two patients, one patient had tourniquet palsy and one complained of persistent pain.

Our complications were similar to study by Javed et al. who had $23 \%$ complication rate with problems such as malunion, persistent pain, stiffness and paresthesia with tingling.

Saw et al. reported four complications with paresthesia, CRPS, AVN and DRUJ instability.

Other studies had similar complications, but Mandalia et al. at the lowest complication rate.

As mentioned there are various fixation options for distal radius fractures. Majority are treated by volar plating, $\mathrm{K}$-wires or an external fixator. Brennan et al. compared volar plating with K-wire fixation where 318 patients were followed up for 32 months, there was no evidence that one had superior functional outcome compared to other (PRWE 17.56 vs. 16.31, $\mathrm{p}=0.69)^{[11]}$. Wilcke et al. showed that outcomes between external fixator and volar plating were similar after one year of follow up (PRWE 11 for external fixator vs. 7 for plating) ${ }^{[12]}$.

Our study in comparison had similar outcomes after 6 months with PRWE score of 16.53 showing fragment specific fixation is an effective option for comminuted intraarticular fractures.

\section{Conclusion}

It is important to use plate according to fracture pattern and according to the need of individual patient; appropriate plate/s can be used. Outcomes are also dependent on adequate and accurate fixation so it can vary with surgical techniques and experience of a surgeon. Fragment specific fixation of distal radius fractures provide good to excellent results and are effective in the correction and maintenance of distal radius anatomy.

\section{References}

1. Jakim I, Pieter HS, Sweet MBE. External fixation for intraarticular fracture of distal radius. J Bone Joint Surg 
(Br). 1991; 73(2):302-306.

2. Colles A. On the fracture of the carpal extremity of the radius. Edinburgh Med Surg. 1814; 10:182-186.

3. Philippe Saffar, William P. Cooney III, editors. Fractures of the distal radius. $1^{\text {st }}$ ed. London: Martin Dunitz, 1995, 136.

4. Leslie BM, Medoff RJ. Fracture specific fixation of distal radius fractures. Tech Orthop. 2000; 15:336-352.

5. Orbay JL, Fernandez DL. Volar fixation for dorsally displaced fractures of the distal radius: a preliminary report. J Hand Surg (Am). 2002; 27:205-215.

6. Barrie KA, Wolfe SW. Internal fixation for intraarticular distal radius fractures. Tech Hand up Extrem Surg. 2002; 6:10-20.

7. Zvi Margaliot, Steven Haase C, Sandra Kotsis V, Myra Kim H, Kevin Chung C. A Meta-Analysis of Outcomes of External Fixation versus Plate Osteosynthesis for Unstable Distal Radius Fractures. The journal of hand surgery. 2005; 30:1185e1-1185e17.

8. Sanjay Gupta, Mansur Halai, Mohammed Al-Maiyah, Scott Muller. Which measure should be used to assess the patient's functional outcome after distal radius fracture? Acta Orthop. Belg. 2014; 80(1):116-118.

9. Saw N, Roberts C, Cutbush K, Hodder M, Couzens G, Ross M. Early experience with the trimed fragment specific fracture fixation system in intra articular distal radius fractures. The journal of hand surgery. 2008; 33(1):53-58.

10. Manthan Mandalia, Sharvil Gajjar, Tailor Anand, Shrey Saxena. Results of distal end radius volar plating. International Journal of Orthopaedics Sciences. 2017; 3(1):395-401.

11. Brennan SA, Kiernan C, Beecher S, OReilly RT, Devitt BM, Kearns SR et al. Volar plate versus k-wire fixation of distal radius fracture. Injury. 2016; 47(2):372-376.

12. Wilcke MKT, Abbaszadegan H, Adolphson PY. Wrist function recovers more rapidly after volar locked plating than after external fixation but the outcomes are similar after 1 year: A randomized study of 63 patients with a dorsally displaced fracture of the distal radius. Acta Orthopaedica. 2011; 82(1):76-81. 